\title{
Assertion, denial, content, and (logical) form
}

\author{
Jack Woods ${ }^{1}$
}

Received: 20 February 2015 / Accepted: 8 June 2015 / Published online: 25 June 2015

(C) Springer Science+Business Media Dordrecht 2015

\begin{abstract}
I discuss Greg Restall's attempt to generate an account of logical consequence from the incoherence of certain packages of assertions and denials. I take up his justification of the cut rule and argue that, in order to avoid counterexamples to cut, he needs, at least, to introduce a notion of logical form. I then suggest a few problems that will arise for his account if a notion of logical form is assumed. I close by sketching what I take to be the most natural minimal way of distinguishing content and form and suggest further problems arising for this route.
\end{abstract}

Keywords Cut/transitivity $\cdot$ Restall $\cdot$ Assertion/denial $\cdot$ Logical form $\cdot$ Logical consequence

Explaining logical consequence in terms of necessary truth-preservation or some variant thereof is very common, but such explanations face difficulties. For one thing, logical consequence seems broader than truth-preservation. Imperatives, for example, can plausibly stand in relations of logical consequence, yet imperatives are neither true or false. Necessary truth preservation, by itself, will thus not explain how we can make logical mistakes in reasoning from imperatives. For another, necessary truthpreservation accounts, even when cashed out in terms of logical form, have difficulty directly satisfying our intuition that a relatively small set of constitutive rules govern the meaning of a logical constant. Why is failing to conform to, e.g. modus ponens, more indicative of a failure to grasp the meaning of the conditional than failing to conform to, e.g. Peirce's law. Third, note that some non-classical logicians, such as

\footnotetext{
$凶$ Jack Woods

j.e.woods@gmail.com

1 Bilkent University, Ankara, Turkey
} 
Hartry Field, have recently argued that validity cannot even imply necessary truthpreservation on pain of paradox (Field 2008). ${ }^{1}$

These and similar frustrations with truth-conditional explanations have lead to a contemporary focus on alternatives formulated directly in terms of correct or permissible inference. ${ }^{2}$ These accounts typically characterize logical consequence in terms of conformity to certain characteristic operational rules, such as modus ponens, which govern the introduction and elimination of logical constants in the context of conformity to certain structural rules which govern more basic features of the consequence relation.

A serious problem with this approach involves saying exactly what the connection between these formal rules and intuitively correct inference is supposed to be. Inference is an action involving changing our beliefs; logical consequence is a relation between (interpreted) sentences, even if generated in terms of formal rules. There is, at least currently, no accepted connection between the action of inference and the relation of logical consequence. ${ }^{3}$ For, as Gilbert Harman has reminded us repeatedly, it is not obviously irrational to refuse to modify our beliefs when they are inconsistent; it is obviously not irrational to fail to draw a consequence of beliefs we antecedently hold, even when we see the connection; and so on. The naive picture of logical consequence as being about correct inference seems, on reflection, simply a mistake. ${ }^{4}$ But it is a seductive mistake and it is hard not to see it as being a bit true. So it would be desirable to have a connection between correct inference and logical consequence; it would be even better if this connection somehow derived from the nature of logical consequence itself.

Greg Restall has recently attempted to make progress on this problem by interpreting logical consequence in terms of a basic notion of rational incoherence which applies to assertions and denials. Logical consequence, at least as captured by the structural rules, is a set of general rational constraints on collections of assertions and denials. Restall's approach is an improvement on the rule-based account in at least two respectsfirst, Harman's worries do not clearly show that there are no constraints on rational coherence which correspond to logical relations; and, second, there is no confusion in Restall's discussion between the action of inferring and the relation of logical consequence. On the assumption of a reasonably strong set of constraints on rational coherence, Restall claims to justify, at a minimum, the standard structural rules of identity, weakening, and cut (Restall 2005). And, moreover, he shows how to extend the account to cover introducing the logical operators as corresponding to other structural features of the consequence relation. ${ }^{5}$

\footnotetext{
1 There are, of course, other putative problems, such as the interpretation of the modal 'necessarily' (Etchemendy 1990). Note that I do not claim that any of these problems is insoluble-far from it. I am here concerned only to motivate why we might look elsewhere for an account of logical consequence.

2 The formal development of such accounts, of course, starts with Gentzen (1934). For a survey of contemporary work and the connection to a philosophical analysis of logical consequence, see Hjortland (2010).

3 See MacFarlane (2004) for a survey of some of the options in this regard.

4 See Harman (1986) for vigorous argument that logic has little to do with inference.

5 More on this below. This way of introducing logical operators continues a project begun in Dosen (1989).
} 
Restall's account also promises to give a satisfying, if partial, account of how logical consequence could be connected with correct inference as well as permissible assertion and denial. If assertion and denial are connected with belief and disbeliefand surely they are-then the fact that it is rationally incoherent to assert and deny $\phi$ plausibly explains why it is incorrect to come to simultaneously believe and disbelieve $\phi$, for any $\phi$. And, finally, Restall's account can plausibly be extended to cover cases like imperatival inference in a fashion similar to how Baker and Woods (2015) handle inconsistency between mental states similar to assertion and denial, it is thus promising as a uniform treatment of logical consequence. ${ }^{6}$

My purpose in the following is to show that Restall's justification of the structural rule of cut fails for the intuitive notion of incoherence governing our assertions unless we assume a notion of logical form, but that assuming a notion of logical form is worrisome for a foundational project of the sort Restall is engaged in. The failure to justify cut is a serious problem-nearly all logics worth taking seriously accept cut. And, moreover, the failure to justify cut undermines Restall's project of giving an ecumenical account of logical consequence which could be accepted by logicians of different stripes. Although I am primarily interested in the virtues of Restall's approach as an explanation of the nature of the consequence relation and logical operations, the demand that Restall's account be formulated in terms of logical form goes through even if we reject this sort of foundational project. Even taken descriptively, we need some account of logical form to make sense of rational incoherence. After criticizing Restall's justification of cut, I will show how to avoid my objection by assuming a very minimal account of logical form and then close by raising a few worries about my solution.

Restall's account uses an intuitive notion of coherence to generate a notion of logical consequence. Coherence is not a mere formal construction; it is rather supposed to be a notion we are at least minimally familar with. Restall expresses this intuitive notion with 'undermines itself' and 'self-defeating' in his general gloss on his project:

In what follows, we will use the notion of a state. Given a particular language which may be rich, containing many different notions, including logical constants, but which may also be completely devoid of any logical constants at all-a state expressed in that language is a pair of sets of statements expressed in that language...Even with this thin notion of state as the focus of our discussion, we can lay down some criteria for evaluating states. Not all states are on a par, for some states are self-defeating. In particular, if a state contains a statement in both the left set and the right set, then this state undermines itself. If the state represents the cognitive architecture of an agent, then this agent both accepts and rejects some statement. If the state represents the state of play in some dialogue or discourse, then some statement has both been asserted and denied. The state is undermined. (Restall 2005, p. 7)

We presumably have a grasp, however loose, of this notion of being selfundermining. And that there are such general features of assertion and denial related to

\footnotetext{
${ }^{6}$ See also our discussion of the sort of explanatory account that is necessary to motivate these types of inconsistency.
} 
incoherence is very plausible - just consider Restall's case of asserting $\phi$ and denying $\phi$. We'll thus grant to Restall that there is some such notion of incoherence attaching to certain states - that is, packagings of assertions and denials — calling it $\perp$. So, $\Gamma \perp \phi$ means “it is incoherent to deny $\phi$ while asserting all of $\Gamma$." Restall's use of $\perp$ is unproblematic given our familiarity with the notion.

Note a couple of additional things about this gloss: first, the languages are unconstrained. Restall is attempting to give an ecumenical picture of the consequence relation. Later remarks indicate that he is attempting to keep this framework neutral between languages like classical logic and those, like dialethist logics, which purport to be universal in the sense that they can contain semantic notions like a truthpredicate. Second, his account of states mentions only the coherence or incoherence of particular packages of assertions and denials in the intuitive sense. Though asserting and denying one and the same statement is one way a state can be incoherent in this intuitive sense, it may not be the only way. In fact, it is not. Thus, nothing said so far guarantees that all intuitive incoherence can be explained in terms of structural features of the consequence relation. We will return to this point below.

One straightforward objection to Restall's account is that the notion of incoherence he uses does not explicate our ordinary notion of logical consequence. As a referee helpfully pointed out, letting $D$ stand for 'is denied', asserting $\neg D \phi$ while denying $\phi$ is intuitively incoherent, but just as intuitively $\neg D \phi$ does not entail $\phi$. If we use this notion of self-undermining to define logical consequence in a language involving operators like $D$, then our account of logical consequence will be slightly revisionary. That is, unless we can find a way to block these examples, we would have to accept that $\neg D \phi$ entails $\phi$, contra our intuitions. I am sympathetic with this sort of objectiontaking such examples to be instances of logical consequences seems to me a significant cost. ${ }^{7}$

Note, though, that being slightly revisionary about the consequence relation is not, by itself, conclusive; many have already worried about the border between logical and semantic consequence, ${ }^{8}$ and the border between semantic and pragmatic consequence is also not entirely clear. ${ }^{9}$ Of course, the example given is not even an example of pragmatic consequence; it simply seems invalid. ${ }^{10}$ And the consequences of the proposed revision are high: $\neg D \phi$ would have as a consequence $\phi$. This would presumably mean that explicitly "prescinding" on $\phi$-asserting that $\phi$ is not denied and that $\phi$ is not asserted-would also commit us to asserting $\phi$. But asserting that $\phi$ is not asserted or denied while asserting $\phi$ seems rationally incoherent since it amounts to asserting that $\phi$ is not asserted (which is one half of prescinding on $\phi$ ) while asserting $\phi$ (a consequence of the other half). The result is that we cannot explicitly prescind

\footnotetext{
7 Ian Rumfitt, in a piece brought to my attention recently, briefly makes a similar objection to Restall's account (Rumfitt 2008). He there argues that 'There is a god' is not entailed by 'There could never be sufficient evidence to accept or deny "There is a god". Again, I am obviously sympathetic. Thanks to Catharine Diehl for discussion of both objections.

8 See Priest (2006, §11.7) and Lycan (1989) for discussion.

9 See Baker and Woods (2015) for an approach to this and initial worries.

10 And, in particular, because we can assert we are not denying something while not asserting it either. Thanks to an anonymous referee for discussion.
} 
on $\phi$ without rational incoherence-a very costly commitment. So, even though the revisionary aspect of Restall's theory is not obviously a decisive reason against it, the costs of the revision are significant enough to worry about this line of defense. But an even more direct problem for Restall's account arises prior to any detailed attempt to explicate our intuitive notion of logical consequence by the relation of incoherence. It arises already for the claim that there is an analogue of the structural rule of Cut for incoherence. We now turn to this problem.

Asserting $\phi$ while simultaneously denying $\phi$ is incoherent in the intuitive sense pointed to above. This feature should remind us of a familiar structural feature of logical consequence: ${ }^{11}$

Identity: $\phi \perp \phi$

Likewise, it just about follows from this that we also get the analogue to the structural rule of left weakening:

Left weakening: $\Gamma \perp \phi \Rightarrow \Gamma, \psi \perp \phi$

since if asserting and denying the package of assertions and denials collected in $\Gamma$ and $\phi$ is incoherent, it ought still be incoherent to assert all of $\Gamma$ and $\psi$ while denying $\phi$. The difficult case is the cut rule. We need:

\section{Cut: $\Gamma \perp \phi$ and $\Delta, \phi \perp \psi \Rightarrow \Gamma, \Delta \perp \psi$}

That is, working with the simplest case:

$$
\Gamma \perp \phi \text { and } \phi \perp \psi \Rightarrow \Gamma \perp \psi
$$

we need that if asserting all of $\Gamma$ while denying $\phi$ is incoherent and if asserting $\phi$ while denying $\psi$ is incoherent, then asserting all of $\Gamma$ while denying $\psi$ is incoherent. Restall motivates this contrapositively by arguing that if we suppose that it's coherent to assert all of $\Gamma$ while denying $\psi$, then it's either coherent to assert all of $\Gamma$ while denying $\phi$ or coherent to assert all of $\Gamma$ and $\phi$ while denying $\psi .^{12}$

It is not immediately obvious why this is true. Restall suggests that there's a suppressed appeal to a version of excluded middle which is plausible for assertion and denial. The idea is that if it's undeniable that $\phi$, then it has to be permissible to assert $\phi$, and, conversely, if $\phi$ cannot coherently be asserted, then it has to be the case that $\phi$ is deniable. However, this is in many ways a strange picture of assertion and denial. Restall's own view is a bit underdescribed:

\footnotetext{
${ }^{11}$ Here and below, I will use 'structural constraints' and 'structural features' as opposed to 'structural rules' since for Restall these are features of the consequence relation, not rules of inference in any reasonable sense.

12 Restall's actual formulation is in terms of multiple conclusions, but showing trouble for the singleconclusion case is sufficient for my purposes.
} 
...the intuitive picture of the connection between assertion and denial. To deny

$A$ is to place it out of further consideration. To go on and to accept $A$ is to change one's mind. Dually, to accept A is to place its denial out of further consideration. (Restall 2005, p. 9)

Nothing about this description rules out the existence of statements on which neither assertion nor denial is warranted; it would be a rather non-ecumenical justification of logic, even granted that only some logics will accept Cut, that required such a strong claim about our intuitive conception of assertion and denial. After all, intuitionistic logic accepts Cut and does not hold that every statement can be asserted or denied, now or even eventually. ${ }^{13}$

Consider, then, a statement $\phi$ which we have no evidence for and no evidence against or, indeed, equally matched evidence for and against. That is, suppose that we do not have evidence which underwrites the assertability or deniability of $\phi$. A plausible picture of assertion and denial has it that proper assertion is underwritten by evidence for the truth of or, at least, a warrant for the statement asserted. And, similarly, proper denial is underwritten by evidence for the falsity, or the counterwarrant for the statement denied. On this account of assertion and denial, it's plausible that there are statements on which the evidence doesn't take a stand.

Now, does this mean that asserting $\phi$ or denying $\phi$ is intuitively incoherent? Not yet, since we can make mistakes about what we have evidence for and evidence against. However, consider the following claims: $\alpha=$ 'there is insufficient evidence to deny $\gamma$ ' and $\beta=$ 'there is sufficient evidence to assert $\gamma$.' It's certainly coherent to assert $\alpha$ and deny $\beta$-this is a package of assertion and denials that amounts to $\gamma$ being something on which we should take no stand. However, consider asserting $\alpha$ and denying $\gamma$. This amounts to a Moorean contradiction, since we've simultaneously denied $\gamma$ while asserting that we have no grounds for denying $\gamma$. Likewise, consider asserting $\gamma$ while denying $\beta$. This amounts to asserting $\gamma$ while denying that we have sufficient evidence to assert $\gamma$. So, even though it's not the case that $\alpha \perp \beta$, it is nevertheless the case that both $\alpha \perp \gamma$ and $\gamma \perp \beta$. So the justification of cut fails-there is a statement $\gamma$ such that both $\alpha \perp \gamma$ and $\gamma \perp \beta$, yet not $\alpha \perp \beta$.

We might think that this notion of denial is too strong - that it would be sufficient to understand denial as (explicitly) withholding assertion. ${ }^{14}$ But this does not help much as we can reformulate the counterexample. Let $\alpha$ be 'there is insufficient reason to withhold on $\gamma$ ' and $\beta$ be 'there is sufficient reason to not withhold on $\gamma$ '. It is clearly unreasonable to assert $\alpha$ while withholding on $\gamma$. It is likewise unreasonable to assert $\gamma$ while explicitly withholding on $\beta$-again, this is pragmatically incoherent. ${ }^{15}$ It is

\footnotetext{
13 Note that Restall is explicit that his background picture does not require commitment to either asserting or denying every statement. But it is difficult to see how to justify Cut without this commitment, as we will see.

14 The 'explicitly' is important here. Withholding has to be an action, otherwise we would conflate taking no stand on something, being lethargic, and having never heard of something.

15 Again, the 'explicitly' is important as there is nothing wrong with asserting $\gamma$ without having heard of $\beta$. We need 'denial' to be something we do, but if it is, then asserting $\gamma$ and denying $\beta$ is incoherent.
} 
perfectly reasonable to assert $\alpha$ and explicitly withhold on $\beta$-this is exactly what we would expect to think of a statement like $\gamma$ which is neither assertible nor deniable. ${ }^{16}$

It might be objected that such constructions involving 'sufficient reason', 'evidence', and reference to statements and sentences aren't fair game, but why not? Given Restall's foundational and ecumenical aspirations, my appeal to notions like evidence, reasons, and names of sentences is unproblematic. ${ }^{17}$ Also note that a similar point can be made with vagueness and borderline cases. Let $F$ be a vague predicate and $a$ firmly in the indefinite region. It is not reasonable to assert $F a$ and it's not reasonable to deny $F a$. That is, it is coherent to assert that it is unreasonable to deny $F a(\alpha)$ and deny that it is reasonable to assert $F a(\beta)$ (not $\alpha \perp \beta$ ) even though both of $\alpha \perp F a$ and $F a \perp \beta$ hold.

Finally, even if we had worries about evidential notions, vagueness, and the like, we can formulate a version of the objection directly. ${ }^{18}$ Let $A \phi$ be ' $\phi$ is asserted' and ' $\neg \mathrm{D} \phi$ ' be ' $\phi$ is not denied'. It is incoherent to assert that $\phi$ is not denied while denying $\phi(\neg D \phi \perp \phi)$. Likewise, it is incoherent to assert $\phi$ while denying that $\phi$ is asserted $(\phi \perp A \phi)$. But it is perfectly coherent to assert that $\phi$ is not denied while denying $\phi$ is asserted $(\neg D \phi \perp A \phi)$.

What this shows is that Restall's justification of Cut relies on the assertions and denials not involving certain forms of content. That is, if we define consequence in Restall's way over a language which includes expressions for, say, assertion and denial, and if we sometimes prescind from doing either, then we lose Cut. ${ }^{19}$ But patterns of incoherence that give the lie to Cut trade on semantic and pragmatic features of content, not general features of assertion and denial (with the notable exception of our last example. But see below).

So if Restall's account is to have any plausibility, we need to be able to isolate the structural features of assertion and denial that hold quite generally, regardless of the content of the assertions involved. This is necessary to distinguish between content or semantic inconsistency which derives from semantic features of what is asserted, and logical inconsistency, which, by Restall's lights, derives from general features of the connection between assertion and denial. The justification of Cut thus relies not just on a general feature of assertion and denial - contrary to the justification of left weakening and identity - but on the content being sufficiently minimal as to prevent rational incoherence deriving from the content of the assertions. So the intended general justification of Cut fails-it is not always the case that if a package of statements is coherently assertible and deniable, in the intuitive sense of coherence, then given an arbitrary additional statement, we can add it to the package of assertions and denials on one side or the other.

There needs to be a way of screening off these cases if Restall is to maintain his claim to be able to recover structural rules like Cut as a feature of a relationship

\footnotetext{
16 I switch here from 'evidence' to 'reason' for purposes of naturalness. Nothing hangs on it.

17 Such attempts at universal logics have become more popular recently. See, for example, Field (2008). One expects that Restall would also want to be able to capture such logics within his framework. And some of these logics, such as Field's, accept Cut.

18 Thanks to a helpful referee for suggesting this direct version of the problem.

19 And mutatis mutandis for all the above examples.
} 
between assertion and denial - in particular, if he is to maintain his further claim that classical logic can be recaptured in this way. This failure should not be surprising. If we adopt the proof-theoretically-oriented formalism Restall works with, it is nearly inevitable to think of schematic letters like $p$ and $q$ as ranging over rather unassuming contents for which the justification will go through without any problem. So written, after all, they look like propositional constants. But our actual assertions and denials range over a far more complex range of content than simple statements. And Restall's notion of incoherence has to do with particular patterns of assertions and denials, not the form of these patterns. ${ }^{20}$

Why, though, should cases of content-based incoherent packages of assertion and denial worry us? Shouldn't our account of consequence be immune to content-based incoherence? The trouble is that we need a way to specify the distinction between form and content. There is a tempting modification of Restall's account which screens off the above cases and thereby allows the justification of the cut rule to go through. What we need is a notion of logical form. Given this, we could reformulate Restall's account in the following way:

$\Gamma \perp \phi$ if and only if it is incoherent to assert all of $\Gamma$ and deny $\phi$ in virtue of the logical form of $\Gamma$ and $\phi .^{21}$

This reformulation avoids the problem I suggested above. But note four important caveats. First, our account of logical form must not presume an account of logical consequence if we are to be giving a non-circular foundational account of logical consequence. Second, this means that an account like Restall's which makes use of assertion and denial to define logical consequence must be formal. This cuts against some of the cases he wants to be able to capture in his framework-such as, for example, the views of folks like Graham Priest who hold that logical consequence is mere necessary truth-preservation. Or, anyways, it does so if he wants to retain cut. ${ }^{22}$

Third, it is rather tempting given Restall's picture to introduce the logical constants as lexicalized features of the consequence relation defined over the structural constraints - that is, as object language terms introduced to correspond to features of the consequence relation. Negation can be interpreted as an object-level indication of moving an item from the assertion chunk of the package to the denial part and vice versa-introduced by transformations like:

\footnotetext{
20 For more on this point, see below.

21 This can be spelled out in a roughly Tarskian fashion even in the context of assertion and denial. Note that capturing the particularly pragmatic aspect of my above examples is harder-see Baker and Woods (2015) for both points in more detail.

22 Nothing I have said here shows that there is not some restricted version of Cut which can be justified in the way Restall suggests. Consider:
}

Relevant Cut: if not: $\Gamma \perp$ and not: $\perp \Delta$, then $\Gamma \perp \phi$ and $\Delta, \phi \perp \psi \Rightarrow \Gamma, \Delta \perp \psi$

That is, if asserting all of $\Gamma$ is not antecedently incoherent, and likewise with denying $\Delta$, then Cut goes through. This is a cut rule reminiscent of Tennant's favored one (Tennant 1987); this restricted cut rule generates a form of relevance logic. But Restall's ambition is greater; he is trying to describe and justify logics far stronger than Tennant's. 


\section{$\Gamma, \phi \perp \psi \Leftrightarrow \Gamma \perp \neg \phi, \psi$}

The conditional is the lexicalization of the $\perp$ relation, sharing some but not all of its features, introduced by some version of the deduction-detachment theorem:

$$
\Gamma, \phi \perp \psi \Leftrightarrow \Gamma \perp \phi \rightarrow \psi
$$

and so on. This would help to explain, as mentioned at the outset, why, intuitively, a small set of constitutive rules (for example, the introduction and elimination transformations for the negation and conditional just given) govern the meaning of the connective. The constitutive rules can be taken as the transformations which introduce the logical constants in question.

But this route is presumably partially closed to us if we proceed by presuming a full account of logical form, including notions like the logical constants, with which we can interpret incoherence in virtue of logical form. After all, if Restall's account is to be foundational in the way described above, then we cannot presume a rich notion of logical form to screen off our examples and introduce the selfsame logical notions as being lexicalized structural features of the consequence relation. ${ }^{23}$ In other words, the worry is that it is a cost to require a foundational account of logic to presume a grasp of the meaning of a privileged set of logical constants in order to avoid the sort of counterexamples above. We need at least some account of logical form in order to avoid the above counterexamples, but it would be unfortunate if presuming this destroyed our hopes of a "bottom-up" account of the logical operators introduced in terms of the structural features of patterns of assertion and denial.

Fourth, and finally, in order to avoid the above counterexamples by use of a form/content distinction, it must be that expressions like 'evidence', 'reasons', 'determinacy', and 'is asserted' are not logical. Otherwise, the above counterexamples could be formulated in purely logical terms, leaving them on the logical side of the divide. ${ }^{24}$ While I would, prima facie, put 'is asserted' on the content side of the form/content divide, this worry is pressing given that assertion and denial correspond to structural feature of the consequence relation-being on the left-hand or right-hand side of the turnstile. And if these terms turn out to be logical, distinguishing form and content will not save Restall's theory as a foundational account. ${ }^{25}$

There are, however, reasons to worry about whether we can introduce an assertion operator by means of transformations like the following:

\footnotetext{
23 Another option is to start off the process of recapturing consequence for a suitably restricted language where contents like the ones above do not appear. The problem is that a general account of consequence would require us to either introduce notions we either introduce notions of evidence, reasonableness, warrant, and the like, which can form part of our basic content, without reintroducing our counterexamples-which is rather implausible; claim that cut holds only for this fragmentary language - an unpalatable result; or somehow build such notions out of a combination of more basic materials and logical consequence in a fashion which does not give rise to our counterexamples - a daunting task.

24 Thanks to a referee for stressing this worry.

25 Note, though, that it is still useful to develop a form/content distinction like the one that I sketch below since we might have reason to use Restall's definition instrumentally in contexts where we do not lexicalize 'is asserted' and 'is denied'.
} 


\section{$\Gamma, \phi \perp \psi \Leftrightarrow \Gamma, A \phi \perp \psi$}

and similarly for denial. It is not as obviously incoherent to assert that $\phi$ is asserted while denying $\phi$ as it is to assert $\phi$ while denying $\phi .{ }^{26}$ But if $A$ is a logical operator governed by the above transformation, then these combinations of assertions and denials should presumably be clearly incoherent given the clear incoherence of $\phi \perp \phi$.

There is also a more indirect worry about the logical status of expressions like 'is asserted'. Since $\phi \perp A \phi$ is intuitively incoherent, given an operator $A$ that obeys the above law, we face the prospect of a truth-like operator (i.e. an operator $A$ such that for all $\phi, A \phi \perp \phi$ and $\phi \perp A \phi)$ — and such operators are prone to paradox, Curry's and otherwise, in the face of self-reference, the structural rules, and a reasonable conditional. ${ }^{27}$ Absent a way of blocking such paradoxes, such operators are maximally non-conservative over our base language. And conservativeness, at least in some form, is often taken to be a necessary condition for an operator to be logical.

So the case for including notions like acceptance as logical operators in our objectlanguage is mixed. On balance, I am agnostic about the logical status and general legitimacy of (unrestricted) terms expressing assertion and denial such as those we might introduce by transformations like the above. ${ }^{28}$ If, as I hope, we can find reasons to deny that assertion and denial (and 'evidence', 'reasons', etc) are logical expressions, then what I give below is a tentative solution to my examples above (modulo my closing worries.) If we cannot, then we have an unresolved objection to Restall's account of logical consequence.

The best way to proceed in screening off my counterexamples to Cut is to try to find the minimal notion of logical form which would suffice to block them yet would not completely foreclose the possibility of introducing logical operators as corresponding to structural features of assertions and denials. An analogous way to put this suggestion is that we need to try to find a minimal way to distinguish between the form and content of a statement. One promising way to do this would be to argue that a notion of generality is built into our understanding of assertion and denial; we could then build on this in order to give a better account of $\perp .{ }^{29}$

\footnotetext{
${ }^{26}$ Note that these worries do not spread to the referee's example of $\neg D \phi \perp \phi$ which is still obviously incoherent-as is its cognate $\phi \perp A \phi$.

27 That is most plausible on the assumption that such operators can be iterated. One might deny this. See Ripley (2014) for a discussion of embedding denial and a useful discussion of paradoxes arising from a denial operator.

28 Worries about the nature and general legitimacy of terms like 'is asserted' and 'is denied' are part of why I have included four counterexamples to Restall's justification of Cut. We need operators occurring in our language, $F$ and $G$, which do not necessarily take a stand on every content $\phi$-i.e. where it is coherent for some content $\phi$ to assert $\neg F \phi$ while denying $G \phi$, but not coherent to assert $\neg F \phi$ while denying $\phi$ nor to assert $\phi$ while denying $G \phi$. That this holds for any of the four can be questioned. But it would be too costly to revise our intuitive grasp of the terms in all four examples (and the many more that could be found.)

29 An interesting alternative account of logical form is articulated in Sagi (2014). On Sagi's view, logical form is a relative notion tied to a more basic type of semantic constraint. There can be pragmatic reasons for choosing what semantic constraints will count as the form of a sentence. The discussion here can be seen as giving a pragmatic reason-the loss of Cut and the collapse of pragmatic and semantic inconsistencyto avoid a too expansive notion of logical form once we accept something like Restall's account as our foundation. Thanks to Nissim Francez for discussion.
} 
So, for example, suppose we started with a simple language with no logical constants, only propositional constants, which we allow to range over any statements whatsoever, such as $\alpha, \beta$, and $\gamma$ above. We presume that we can generalize over propositional constants $\mathrm{s}^{30}$ and have an intuitive notion of conjunction allowing us to "jointly assert" and "jointly deny". ${ }^{31}$ We can then read $\Gamma \perp \Delta$ as "it is incoherent to assert all of $\Gamma$ and deny all of $\Delta .{ }^{.32}$ More formally, given Restall's notion of incoherence, we can use these two features to generate a better notion of incoherence. We define it as follows:

$$
\psi_{0}, \ldots, \psi_{n} \pm \phi_{n+1}, \ldots \phi_{j} \text { if and only if } \forall p_{0} \ldots p_{k}\left[\psi_{0}, \ldots, \psi_{n} \perp \phi_{n+1}, \ldots \phi_{j}\right]
$$

where

$$
\forall p_{0} \ldots p_{k}\left[\psi_{0}, \ldots, \psi_{n} \perp \phi_{n+1}, \ldots \phi_{j}\right]
$$

is the universal closure of

$$
\left[\psi_{0}, \ldots, \psi_{n} \perp \phi_{n+1}, \ldots \phi_{j}\right]
$$

in the obvious sense. For example, $\left\{p_{0}, p_{1}, p_{2}\right\} \pm p_{0}$ since $\forall p_{0}, p_{1}, p_{2}\left\{p_{0}, p_{1}, p_{2}\right\} \perp p_{0}$. That is, because for all propositional contents, the package of assertion and denials where something is both asserted and denied is incoherent-no matter what it is.

We can now capture the structural constraints as Restall does, using \pm instead of $\perp$; note that my examples fail since none of the cited patterns is incoherent for all ways of uniformly assigning propositional contents to propositional constants. The justification of Cut now has an interesting property (for our restricted language): anytime $\Gamma \pm \phi, \phi$ occurs in $\Gamma$. And, in general, if $\Gamma \pm \Delta$, then $\Gamma$ and $\Delta$ are not disjoint. So, if $\Gamma \pm \phi$ and $\Delta, \phi \pm \psi$, then $\psi$ must occur in $\Delta$ or be identical to $\phi$-and, either way, $\psi$ occurs in $\Gamma \cup \Delta$. In effect, this method reduces the justification of Cut to the justification of identity, making it analogous to the justification of left weakening. This is what we want-in the context of a minimal language with no logical constants, incoherence of pattern should really reduce to general features of the assertion/denial relation and the only such feature available when we generalize over the content is the repetition of a statement.

\footnotetext{
30 This is an expository simplification. A fully rigorous treatment would distinguish, say, between propositional constants and propositional variables. But, as such technicalities would distract here and the point is clear enough, I simply take quantification over propositional constants for granted.

31 Conjunction is about the most reasonable logical concept to take for granted. It is singularly well-behaved: for example, the rules for introduction and elimination of conjunctions uniquely specify the meaning on any way of defining the connection between proof rules and meanings. It is the only non-trivial constant having this property. It is so close to fundamental to understanding assertion that John Stuart Mill once quipped that to call a conjunction a compound proposition was tantamount to calling a street a compound house.

32 Note that this is to read the structural comma as conjunctive on both sides. One of Restall's nice points is noticing that in the presence of a basic notion of denial, we do not need to read the structural comma on the right disjunctively. This avoids many problems, not least of which is that alternation does not seem to be a basic feature of assertions and denials.
} 
Note that logical constants such as a sign for negation appear nowhere in our fragmentary language. We can introduce it and like constants in a similar fashion to Restall. Negation can be modeled as the lexical item corresponding to moving a statement from the left of \pm to the right and conversely. So:

$$
\begin{aligned}
& \Gamma, \phi \pm \psi \Leftrightarrow \Gamma \pm \neg \phi, \psi \\
& \Gamma \pm \phi, \psi \Leftrightarrow \Gamma, \neg \phi \pm \psi
\end{aligned}
$$

We can then check that the structural properties are preserved under this stipulationthe proof is tedious but routine-and likewise for the other logical constants. In the presence of quantification over statements and a suppressed notion of conjunction, then, we can recover facsimiles of the logical constants and a recognizable consequence relation, here given by \pm . There remain a pair of difficult questions for this approach, taken foundationally.

First, and most importantly, what guarantees the defined notions correspond to our intuitive grasp of the logical constants? This problem comes in two guises-first, and less importantly, note that $\pm \phi, \neg \phi$ is derivable immediately from our stipulated definition of $\neg$ and the structural rule of identity. But this means that it is always rationally incoherent to deny both something and its negation. This is both tempting and by no means obvious. Once we get the full account of logical complexity, we can formulate pairs of statements which are very plausibly jointly deniable, even though one is logically equivalent to the negation of the other (Harman 1986). Since the structural constraints have been justified in a satisfying way, the problem is with the stipulated conditions for the classical logical constants in the context of assertion and denial. In some way, these stipulated pseudo-constants must come apart from the intuitive notions which we set out to formalize or, alternatively, we must accept that we are merely defining pseudo-constants with a hyper-idealized notion of rational coherence. But, if we take the latter tack, we need to describe how our intuitive conception of the logical constants corresponds to these notions defined in this way since we, of course, do not have an intuitive grasp of this hyper-idealized notion of logical coherence. ${ }^{33}$ Note that no analogue problem arises for ordinary Tarskian accounts of logical consequence. On that account, logical consequence is one thing, what it is coherent to assert and deny quite another. ${ }^{34}$

Second, we need to justify the use of generality - in particular, quantification over statements, or, more generally, contents - as a feature of our grasp of assertion and denial. At a minimum, the notion of generality has to be grasped antecedently to our stipulations of logical constants. Given that a notion of generality requires a grasp of

\footnotetext{
33 The problem is not that we cannot generate this idealized notion out of assertion, denial, definitions, and quantification over statements. We can. Rather, the problem is that the notion generated in this way diverges from our intuitive grasp of the constants since it is intuitively not incoherent to believe inconsistencies.

34 Note that I am not claiming that no similar problem arises for the Tarskian account conjoined with other theses about assertion and belief. Just that the Tarskian account by itself is insufficient as a source of this kind of problem. Of course, the Tarskian account, like any formal representation of an intuitive notion, faces the distinct problem of explaining how the formal account represents the intuitive one. See Sher (1991) for a useful formal and philosophical overview of the Tarskian approach and Hanson (2002) for some worries about Sher's approach.
} 
sameness and difference for statements - that is, a criterion of identity of statementsit is not obvious how this will proceed in a way which allows our grasp of logic to come after our grasp of assertion and denial. ${ }^{35}$ If we are working in a very impoverished language, this problem is reduced-we need only presume that we have a grasp of when we are asserting and denying one and the same statement. But, again, we ordinary thinkers work with a language of far more complexity and far less univocality. How the defined notion relates back to our grasp of logic is an open question. ${ }^{36}$

I have argued that Restall's justification of the structural features of logical consequence in terms of incoherence of certain combinations of assertions and denials is subject to counterexamples. If the content we assert and deny includes notions like 'sufficient evidence to assert/deny' which attach to other contents, and if, say, evidence doesn't decide on every content, then there are cases which violate Cut. Restall's justification only goes through when we presume some notion of logical form. A notion of logical form allows us to narrow the relevant notion of incoherence to incoherence in virtue of logical form, blocking counterexamples to Cut involving expressions like 'sufficient evidence' and so on which are plausibly non-logical. ${ }^{37}$ I then showed how a particular minimalistic version of this involving quantification over statements and conjunction would work. I closed by questioning the reasonableness of taking an account like this foundational. That is, to be plausible as any sort of account of logical consequence, Restall's picture needs a notion of logical form-even if a rather minimal one. But it is by no means obvious that when you add an account of logical form it remains as tempting as a foundational account of our grasp of logic.

Acknowledgments Thanks to Catharine Diehl, Nissim Francez, Nick Stang, and two anonymous (and helpful!) referees for useful discussion. Thanks also to John Burgess for remarks on a distant ancestor of this paper and Jimmy Martin for a conversation about it that I reckon he's forgotten.

\section{References}

Baker, D., \& Woods, J. (2015). How expressivists can and should explain inconsistency. Ethics, 125(2), 391-424.

Dosen, K. (1989). Logical constants as punctuation marks. Notre Dame Journal of Formal Logic, 30(3), $362-381$.

Etchemendy, J. (1990). The concept of logical consequence. Cambridge: Harvard University Press.

Field, H. (2008). Saving truth from paradox. Oxford: Oxford University Press.

Garson, J. W. (2013). What logic means: From proof theory to model-theoretic semantics. Cambridge: Cambridge University Press.

Gentzen, G. (1934). Untersuchungen über das logische Schliessen. Mathematische Zeitschrift, 39, 176-210. Hanson, W. H. (2002). The formal-structural view of logical consequence: A reply to Gila Sher. The Philosophical Review, 111(2), 243-258.

\footnotetext{
35 This problem is related, of course, to the sort of worry that Quine raises against Carnap in Quine (1936). Quine's point is that you need logic to get logic from definitions. Our point is that you may need logic to understand how we can quantify over statements in such a way as to recognize patterns of any old form.

36 Note, in defense of this approach, that attempting to give a rule-based justification of quantification or generality is quite difficult; see Garson (2013) for details in the case of model-theoretic inferentialism. Similar worries apply to Restall's sort of approach.

37 The logical status of 'is asserted' is less clear. If such expressions are logical, then Restall's account faces more troubles than my simple form/content distinction can finesse. See above discussion.
} 
Harman, G. (1986). Change in view. Cambridge: MIT Press.

Hjortland, O. T. (2010). The structure of logical consequence: Proof-theoretic conceptions. Ph.D. Thesis, University of St. Andrews

Lycan, W. G. (1989). Logical constants and the glory of truth-conditional semantics. Notre Dame Journal of Formal Logic, 30(3), 390-400.

MacFarlane, J. (2004). In what sense (if any) is logic normative for thought. Presented at American Philosophical Association Central Division meeting.

Priest, G. (2006). Doubt truth to be a liar. Oxford: Oxford University Press.

Quine, W. V. O. (1936). Truth by convention. In O. H. Lee (Ed.), Philosophical essays for A. N. Whitehead (90-124). London: Longmans, Green \& Co.

Restall, G. (2005). Multiple conclusions. In P. Hájek, L. Valdes Villanueva, \& D. Westerståhl (Eds.), Logic, methodology and philosophy of science: Proceedings of the twelfth international congress (189-205). London: Kings College Publications.

Ripley, D. (2014). Embedding denial. In O. T. Hjortland \& C. Caret (Eds.), Foundations of logical consequence. Oxford: Oxford University Press.

Rumfitt, I. (2008). Knowledge by deduction. Grazer Philosophische Studien, 77(1), 61-84.

Sagi, G. (2014). Formality in logic: From logical terms to semantic constraints. Logique et Analyse, 227, 259-276.

Sher, G. (1991). The bounds of logic: A generalized viewpoint. Cambridge: MIT Press.

Tennant, N. (1987). Anti-realism and logic: Truth as eternal. Oxford: Clarendon Press. 